\title{
Proactive Interference as a Result of Persisting Neural Representations of Previously Learned Motor Skills in Primary Motor Cortex
}

\author{
Nicholas Cothros, Stefan Köhler, Erin W. Dickie*, \\ Seyed M. Mirsattari, and Paul L. Gribble
}

\begin{abstract}
Learning to control movements in different dynamic environments is marked by proactive interference; learning a first skill interferes with the subsequent learning of a second one. The neural basis of this effect is poorly understood. We tested the idea that proactive interference results from persisting neural representations of previously learned skills in the primary motor cortex (M1). We used repetitive transcranial magnetic stimulation (rTMS) of M1 to disrupt retention of a recently learned
\end{abstract}

\section{INTRODUCTION}

The primate motor system can produce accurate movements under a variety of mechanical conditions as a result of motor learning. This ability is thought to be based on the acquisition of neural representations of the mechanical requirements of movement (Gribble \& Scott, 2002; Gandolfo, Li, Benda, Schioppa, \& Bizzi, 2000; Conditt, Gandolfo, \& Mussa-Ivaldi, 1997; Flanagan \& Wing, 1997; Brashers-Krug, Shadmehr, \& Bizzi, 1996; Shadmehr \& Mussa-Ivaldi, 1994). There is a body of evidence that implicates the primary motor cortex (M1) as part of a network of brain regions involved in learning and retention of motor skills. Electrophysiological recordings of M1 in nonhuman primates have identified a population of M1 cells that appear to underlie motor memory after adaptation to novel dynamics (Li, Padoa-Schioppa, \& Bizzi, 2001; Gandolfo et al., 2000). Neuroimaging studies in humans have shown that extensive practice of a finger-tapping task results in a progressive enlargement of its representation in M1 (Karni et al., 1998; Karni et al., 1995), and other studies using transcranial magnetic stimulation (TMS) have shown that M1 is reorganized following motor learning (Classen, Liepert, Wise, Hallett, \& Cohen, 1998).

University of Western Ontario, Canada

* Present address: Brain Imaging Group, Douglas Hospital Research Centre, McGill University, Montréal, Québec, Canada $\mathrm{H} 4 \mathrm{H} 1 \mathrm{R} 3$. motor skill. If interference results from the retention of this skill then its disruption should be associated with reduced interference. Subjects reached to targets while interacting with a robotic arm that applied force fields to the limb. Fifteen minutes of $1-\mathrm{Hz}$ rTMS to M1 impaired the retention of a first force field, and more importantly, reduced proactive interference when subjects learned a second one. Our findings suggest that retention and interference are linked at the level of M1.

Behavioral studies have shown that learning different motor skills in close temporal proximity results in interference (Brashers-Krug et al., 1996). Proactive interference occurs when the effects of learning one skill result in decreased performance when learning a subsequent skill. Neuroimaging and electromyographic studies have shown that the physiological correlates of motor learning persist when subjects are subsequently exposed to different dynamics (Thoroughman \& Shadmehr, 1999; Shadmehr \& Brashers-Krug, 1997; Shadmehr \& Holcomb, 1997). It has been proposed that interference may result from competing neural representations in motor working memory (Wigmore, Tong, \& Flanagan, 2002). However, the neural basis of interference and its relationship to the retention of recently learned motor skills is currently unclear.

In this study we tested the idea that proactive interference results from persisting neural representations of previously learned motor skills in M1. We used lowfrequency repetitive transcranial magnetic stimulation (rTMS) to temporarily disrupt neural processing in M1 (Pascual-Leone, Bartres-Faz, \& Keenan, 1999). We employed a motor learning task in which subjects reached to targets while interacting with a robotic arm that applied two different force fields to the limb (see Methods). Following the learning of one force field, a 15-min 1-Hz rTMS train was delivered to M1, contralateral to the moving limb. Subjects were then either presented with the same force field, or were asked to learn a different one. If information about how to 
control the limb in the first force field is represented in M1, then disruption of this representation using rTMS should impair retention (see Muellbacher et al., 2002, for a related finding). In addition, if proactive interference results from the effects of persisting neural representations of a previously learned force field, then disruption of the representation of the first force field should reduce interference. In light of proposals that interference effects are time limited (Shadmehr \& Brashers-Krug, 1997), we examined the role of M1 both immediately after learning and after a 24-hr delay.

\section{METHODS}

\section{Subjects}

A total of 61 subjects participated in the study (see Table 1). All subjects were right-handed and were randomly assigned to one of eight groups. All subjects reported normal or corrected-to-normal vision, no history of neurological or musculoskeletal disorder, and gave their written informed consent before participation. Subjects receiving rTMS were screened for standard exclusion criteria for TMS and magnetic resonance imaging (MRI) (Wassermann et al., 1996). The University of Western Ontario Research Ethics Board approved all procedures. No subjects participated in more than one condition.

\section{Transcranial Magnetic Stimulation}

All subjects receiving TMS underwent a high-resolution anatomical MRI scan in a 4-T Siemens-Varian scanner. The MR image was used to guide spatially accurate delivery of TMS pulses. Each subject's MRI scan was coregistered with the subject's head position using Brainsight software (Rogue Research, Montréal, Canada) and the Polaris infrared motion tracking device (Northern Digital, Waterloo, Canada), based on nine landmarks on the head. Five of these landmarks corresponded to the location of vitamin $\mathrm{E}$ capsules, fixed to the scalp for the duration of the MRI scan. The remaining four landmarks were anatomical features, namely, the bridge and tip of the nose, and the junction of the crus and tragus in each ear. Subjects' heads were stabilized in a chin rest. Following coregistration, the Polaris system was used to position the TMS coil such that the direction of induced current in the brain flowed in a posteroanterior direction, roughly perpendicular to the central sulcus. This orientation has been shown to preferentially excite M1 interneurons rather than direct activation of the corticospinal tract (see Di Lazzaro et al., 2004, for a review). A cursor on the computer screen indicated the real-time position of the TMS coil over the cortex as it was positioned over the subject's scalp.

TMS pulses were delivered by using an air-cooled figure-of-eight coil, facilitated by a biphasic rapid-rate

Table 1. Summary of Experimental Groups

\begin{tabular}{|c|c|c|c|c|c|}
\hline & \multirow[b]{2}{*}{ Group } & \multicolumn{3}{|c|}{ Experimental Protocol } & \multirow[b]{2}{*}{$N$} \\
\hline & & Force Field 1 & Delay & Force Field 2 & \\
\hline \multirow[t]{4}{*}{ Effects of rTMS M1 on proactive interference } & A1 & $\mathrm{CW}$ & $30 \mathrm{~min}$ & $\mathrm{CCW}$ & 7 \\
\hline & $\mathrm{A} 2$ & NULL & $30 \mathrm{~min}$ & $\mathrm{CCW}$ & 7 \\
\hline & A3 & $\mathrm{CW}$ & $30 \mathrm{~min}$ & $\mathrm{CCW}$ & 6 \\
\hline & & & 15 min rTMS M1 & & \\
\hline \multirow[t]{3}{*}{ Time course } & B1 & $\mathrm{CW}$ & $24 \mathrm{hr}$ & $\mathrm{CCW}$ & 9 \\
\hline & & & 15 min rTMS M1 & & \\
\hline & $\mathrm{B} 2$ & $\mathrm{CW}$ & $24 \mathrm{hr}$ & $\mathrm{CCW}$ & 6 \\
\hline \multirow[t]{3}{*}{ Effects of rTMS M1 on retention } & $\mathrm{C} 1$ & $\mathrm{CCW}$ & $30 \mathrm{~min}$ & $\mathrm{CCW}$ & 7 \\
\hline & $\mathrm{C} 2$ & $\mathrm{CCW}$ & $30 \mathrm{~min}$ & $\mathrm{CCW}$ & 5 \\
\hline & & & 15 min rTMS M1 & & \\
\hline \multirow[t]{4}{*}{ Control for nonspecific effects } & D1 & $\mathrm{CW}$ & $30 \mathrm{~min}$ & $\mathrm{CCW}$ & 6 \\
\hline & & & 15 min rTMS SFG & & \\
\hline & D2 & $\mathrm{CCW}$ & $30 \mathrm{~min}$ & $\mathrm{CCW}$ & 4 \\
\hline & & & 15 min rTMS SFG & & \\
\hline \multirow[t]{2}{*}{ Effects of rTMS on learning rate } & $\mathrm{E} 1$ & NULL & $30 \mathrm{~min}$ & $\mathrm{CCW}$ & 4 \\
\hline & & & 15 min rTMS M1 & & \\
\hline
\end{tabular}


stimulator (Magstim). The intensity of stimulation of the rTMS train was $110 \%$ of the resting motor threshold. Resting motor threshold was defined as the intensity at which a motor-evoked potential (MEP) could be reliably elicited from the right first dorsal interosseus and/or the right extensor carpi radialis, through single TMS pulses delivered to contralateral (left) M1. This method represents the current convention in the field and in particular was important in maintaining comparability with a related study of the effects of rTMS on motor learning (Muellbacher et al., 2002). Thresholds for more proximal muscles, for example, biceps brachii, were typically 5$10 \%$ higher than those for hand and wrist muscles. Two monopolar electromyograph (EMG) electrodes were attached to the skin over each of four muscles of the right arm: middle deltoid, biceps longhead, extensor carpi radialis, and first dorsal interosseus. MEPs were visualized on a computerized oscilloscope. After finding resting motor threshold, further single pulses were delivered at $110 \%$ in order to find the M1 representation of deltoid and biceps muscles. Stimulation was directed between two targets corresponding to the representations of deltoid and biceps. This site of stimulation was chosen based on a previously reported protocol in which stimulation was aimed at the representation of muscles that are used chiefly in the motor task (Muellbacher et al., 2002). For the group that received rTMS delivered to the contralateral (left) superior frontal gyrus, the site of stimulation was located according to the following protocol: First, on the transverse view of the two-dimensional MRI slices, the left insula was located. From a point near the insula that lies on the outermost cortical surface of the cerebrum, an imaginary line running in the dorsal/ventral direction, perpendicular to the sylvian sulcus was established. This line was extended to the superior frontal gyrus, where rTMS pulses were delivered.

The duration of the rTMS train was $15 \mathrm{~min}$, at a rate of $1 \mathrm{~Hz}$ and at an intensity of $110 \%$ resting motor threshold. The mean threshold for distal muscles was $55.24 \%$ of maximum stimulator output $(S D=4.95)$. The maximum intensity used was $70 \%$ of maximum stimulator output. Subjects were instructed to sit still in the stereotaxy during rTMS, with their chins in a chin rest and limbs at rest. The TMS coil, which was attached to a multilink adjustable arm, was held in place on the subjects' scalp.

\section{Motor Learning Task}

The motor learning task entailed making reaching movements while gripping an InMotion2 (InMotion Technologies, Cambridge, MA) robotic manipulandum in the right hand (Figure 1A). A custom air sled resting on a table supported the right upper arm during the task. The air sled carried a cushion of medium-density tempur foam (Kees Goebel Medical) to cradle the upper arm. Drawing from a 40-psi compressed air source, the air

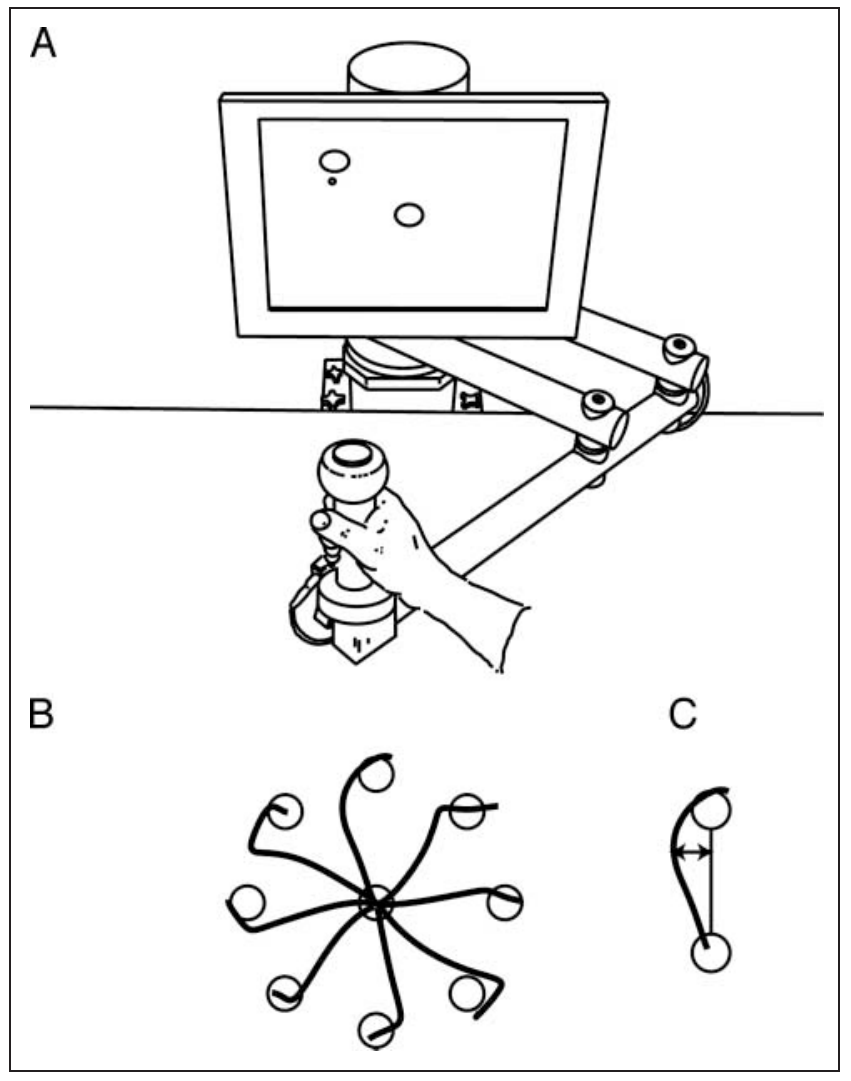

Figure 1. Experimental task. (A) Subjects were instructed to move the end of a robotic manipulandum in a horizontal plane. Movement targets and a cursor representing the location of the hand were displayed on a computer screen in real time. The robotic manipulandum produced forces that acted at the hand, proportional to the velocity of movement. Force fields acted in either a clockwise (CW) or counterclockwise (CCW) direction. (B) Typical reaching movements during early exposure to a CCW force field. (C) Movement curvature was used as a measure of performance and was quantified using perpendicular distance (PD), that is, the maximum orthogonal deviation between the hand and the line segment connecting the central start position and the target.

sled directed airflow directly beneath the sled, creating a cushion of air between the sled and the table, thereby reducing friction and supporting the arm against gravity. Subjects made movements in a horizontal plane, involving primarily shoulder and elbow rotation. Reaching movements were made toward eight targets surrounding a central start position at a constant radial distance $(10 \mathrm{~cm})$ and at $45^{\circ}$ intervals (Figure 1B). The position of the hand was represented in real time by a cursor on a screen directly in front of the subject. Starting from the central start position, a target appeared on the periphery of the screen. The task was to make a brisk, accurate reaching movement to the target within a limited time window (420-520 msec). When the target was reached, it changed color to indicate movement speed. Red denoted a movement that was too fast, green denoted a movement that was too slow, and yellow indicated a 
movement that was made at the appropriate speed. Subjects were instructed to adjust movement speed so as to avoid red and green signals. The disappearance of the target served as a cue to return to the central start position, after which another target would appear. Movement initiation was defined as the first time the tangential velocity of the hand exceeded $0.05 \mathrm{~m} / \mathrm{sec}$. Movement termination was defined as the first time the tangential velocity of the hand fell below $0.05 \mathrm{~m} / \mathrm{sec}$ after movement initiation.

The robotic manipulandum produced forces that acted at the hand, proportional to the velocity of movement. These forces acted either in a clockwise (CW) or counterclockwise (CCW) direction, depending on the phase of the experiment. The forces were defined by the following equation:

$$
\left[\begin{array}{c}
F_{x} \\
F_{y}
\end{array}\right]=\left[\begin{array}{cc}
0 & d k \\
-d k & 0
\end{array}\right]\left[\begin{array}{l}
x \\
y
\end{array}\right]
$$

where $F_{x}$ and $F_{y}$ are forces generated by the manipulandum in the left/right and forward/backward directions, respectively, $x$ and $y$ denote hand velocities, $k=$ $20 \mathrm{~N} \mathrm{sec} / \mathrm{m}$, and $d=+1.0(\mathrm{CW})$ or $d=-1.0(\mathrm{CCW})$. In some experimental conditions, the manipulandum produced no forces (a null field). Limb positions, velocities, and forces were recorded at $500 \mathrm{~Hz}$ and stored on a computer.

\section{Procedure}

All subjects initially interacted with the robotic manipulandum in one type of force field. Following a 30-min delay, subjects returned to the manipulandum and were exposed to a new field. The two fields each consisted of three blocks of 192 movements. Prior to the first field, all subjects performed the task in a null field (NULL), where no force field was present, providing a period of habituation. A total of 192 movements were made in NULL. Thus, all subjects performed seven blocks of reaching movements. The presentation of targets was pseudorandomized over blocks of eight movements. This eighttarget cycle was repeated 24 times per block, for a total of 192 movements per block.

\section{Data Analysis}

All data analyses were conducted using MATLAB software (The Mathworks Inc.) and SPSS software (SPSS Inc., Chicago, IL) Perpendicular distance (PD) was defined as the maximum orthogonal deviation between the hand and the line segment defined by the central start position and the target in question. PD thus provides a measure of movement perturbation. Positive values for
PD indicated a deviation in the CCW direction and negative values indicated a CW deviation. Three other measures of movement performance were calculated for each movement: peak hand tangential velocity, time to peak hand tangential velocity, and to assess movement smoothness, hand trajectory root-mean-squared jerk. For each of the dependent variables, mean values were computed over successive windows of eight movements. To assess initial performance when learning a force field, means were calculated over the first 16 movements. Differences between groups were tested using betweensubjects analysis of variance (ANOVA) and independent samples $t$ tests. Tukey tests were used for post hoc comparisons among individual means.

\section{RESULTS}

Subjects were instructed to make reaching movements while gripping a robotic manipulandum in the right hand (Figure 1A). Reaching movements were made toward eight targets, surrounding a central start position at a constant radial distance and at $45^{\circ}$ intervals (see Methods, Figure 1B). The task was to make a brisk, accurate reaching movement to the target within a limited time window. The manipulandum produced forces that acted at the hand in proportion to the tangential velocity of the endpoint. Depending on the phase of the experiment, the manipulandum produced a CW force field (Methods) or a CCW force field. Subjects interacted with the manipulandum in one type of force field, and following a 30-min delay, subjects returned to the manipulandum and were exposed to a new field. Performance in the reaching task was characterized by measuring the curvature of the movement trajectory using PD, the maximum orthogonal deviation between the hand and the line segment connecting the central start position and the target (see Figure 1C). PD reflects a subject's skill in properly compensating for the perturbing effects of a force field. As this skill increases, movement curvature and, hence, PD decreases.

\section{Effects of rTMS over M1 on Interference}

Three groups of subjects were compared in this first study (see Table 1). One group (A1) first learned CW, and then following a 30-min delay learned CCW. A second group (A2) did not learn CW but performed an equal number of movements without any forces (NULL), and following a 30-min delay learned CCW. By comparing these two groups, we can assess proactive interference in our paradigm. To explore the role of M1 in proactive interference, a third group (A3) learned CW, and during the 30-min delay underwent rTMS delivered to contralateral M1 (see Methods), and then learned CCW. Differences in initial movement performance among the three groups were tested by measuring 
movement curvature over the first 16 movements in CCW. Between-subjects ANOVA was used to test for differences in PD. Tukey post hoc tests were used to assess differences among individual means.

Performance of subjects that had previously learned CW (A1) was significantly worse (i.e., movement curvature was greater) than that of subjects who were not exposed to CW prior to learning CCW (A2) $(p<.05$; Figure 2A). This decrease in performance reflects the effects of proactive interference (Shadmehr \& BrashersKrug, 1997; Brashers-Krug et al., 1996). In contrast, initial performance in CCW of subjects who received rTMS to M1 after learning CW (A3) was significantly greater (i.e., movement curvature was reduced) than that of subjects who learned the same force fields but who did not receive rTMS (A1) $(p<.05$; Figures 2A and $3 \mathrm{~A}$ ). This increase in performance reflects a decrease in the magnitude of proactive interference, indicating a critical role of M1 in this process. Nota-

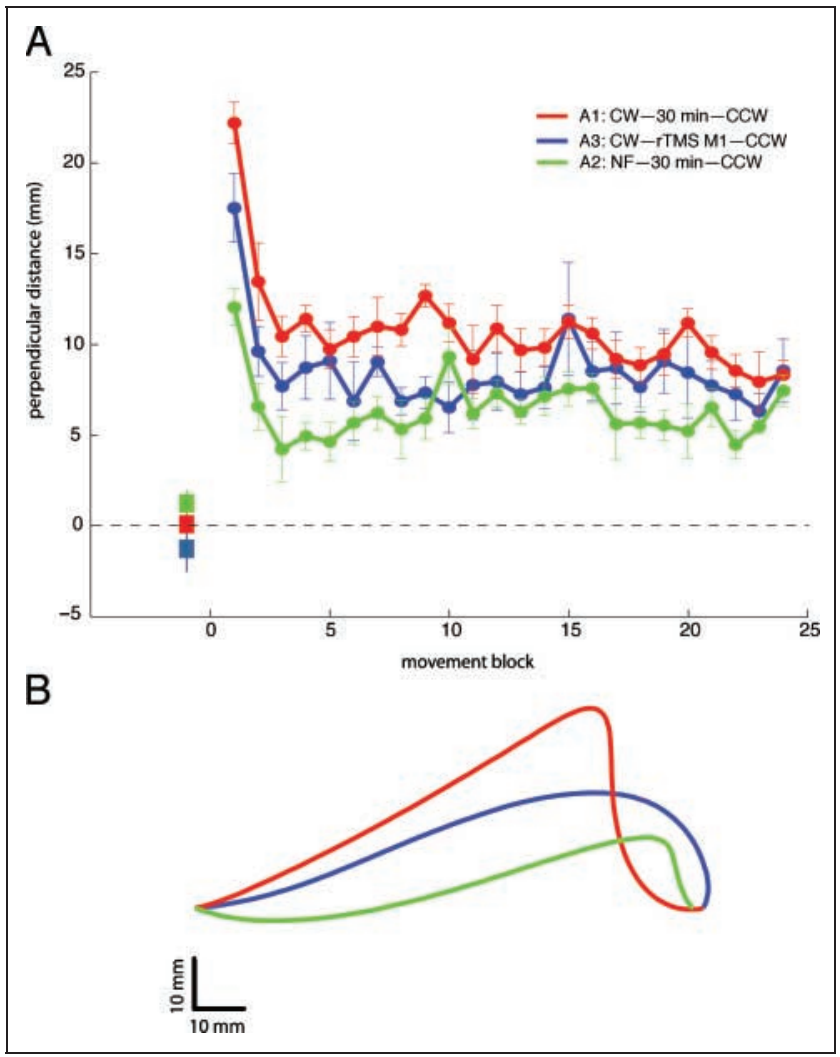

Figure 2. Effects of rTMS on proactive interference. Performance in CCW for subjects who previously learned CW (red), subjects with no previous exposure to a force field (green), and subjects who previously learned $\mathrm{CW}$ and received rTMS prior to CCW (blue). (A) Each point denotes the mean PD over eight movements. Vertical bars correspond to one standard error of the mean. Squares indicate mean PD over the last 16 movements prior to exposure to CCW. (B) Hand path trajectories for the initial movement in CCW for three single subjects. Each trace corresponds to a single movement trajectory from left to right. The traces are organized such that the beginning and end of the movements are aligned horizontally.

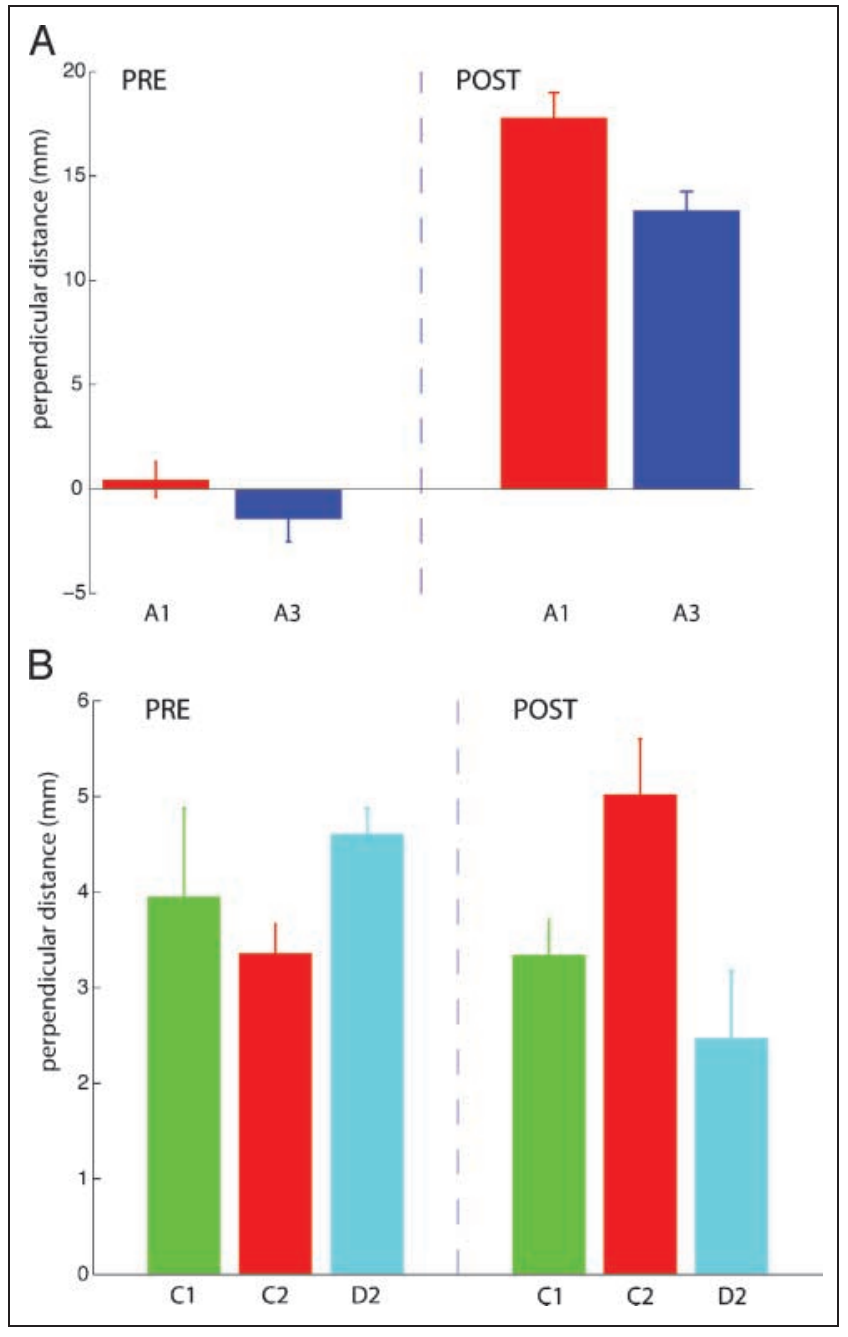

Figure 3. Differential effects of rTMS depending on previous learning experience. Mean PD across the last 16 movements prior to the 30-min delay (PRE) and the first 16 movements in CCW following the 30-min delay (POST) is shown. Vertical bars denote one standard error of the mean. (A) When the force field preceding rTMS (CW) was different from the one presented after stimulation $(\mathrm{CCW})$, subjects showed an improvement in performance (i.e., reduced proactive interference, blue) relative to the corresponding controls who did not receive rTMS (red). (B) In contrast, when subjects were exposed to the same force field (CCW) before and after rTMS, a decrease in performance (i.e., an increase in curvature, red) was observed relative to controls who did not receive rTMS (green). Subjects who were exposed to the same force fields and received rTMS over the superior frontal gyrus (SFG) did not show a disruption in performance (cyan).

bly, although proactive interference was reduced by rTMS to M1, it was not eliminated entirely. Performance of subjects who received rTMS (A2) was still significantly worse than that of subjects who were not exposed to CW before learning CCW (A2) $(p<.05$; Figure $2 \mathrm{~A}$ ). The reduction in proactive interference following rTMS to M1 was observed in the initial movement trajectories in CCW, which showed less curvature relative to subjects who learned the same forces but did 
not receive rTMS. The amount of movement curvature in CCW, however, was greater than that of subjects who were not exposed to $\mathrm{CW}$ prior to learning CCW (see Figure 2B).

To rule out the possibility that the observed effects of rTMS were due to differences in performance prior to exposure to CCW, we also tested for differences between mean PD in the last 16 trials prior to CCW between the three groups (A1, A2, A3). No significant differences were observed ( $p=.11$, between-subjects ANOVA; Figure 2A).

\section{Time Course of Effects of rTMS}

To examine the time course of the effects of rTMS applied to M1 on proactive interference, we tested an additional group of subjects (B1) who received rTMS $24 \mathrm{hr}$ after learning $\mathrm{CW}$, just prior to learning CCW. Initial performance in CCW (mean PD over the first 16 movements in CCW) in this group was not significantly different from performance of subjects who received rTMS after $30 \mathrm{~min}$ (A3, above; $p=.63$, independent samples $t$ test; Figure 4 ). Thus, a reduction of proactive interference following rTMS to M1 was observed regardless of the delay separating the two force fields. This suggests that the information resulting from the learning of CW was still in a labile form after $24 \mathrm{hr}$. In other words, this information was not consolidated. To rule out the possibility that this effect may have been due to differences in performance prior to CCW, we also tested for differences between mean $\mathrm{PD}$ in the last 16 trials

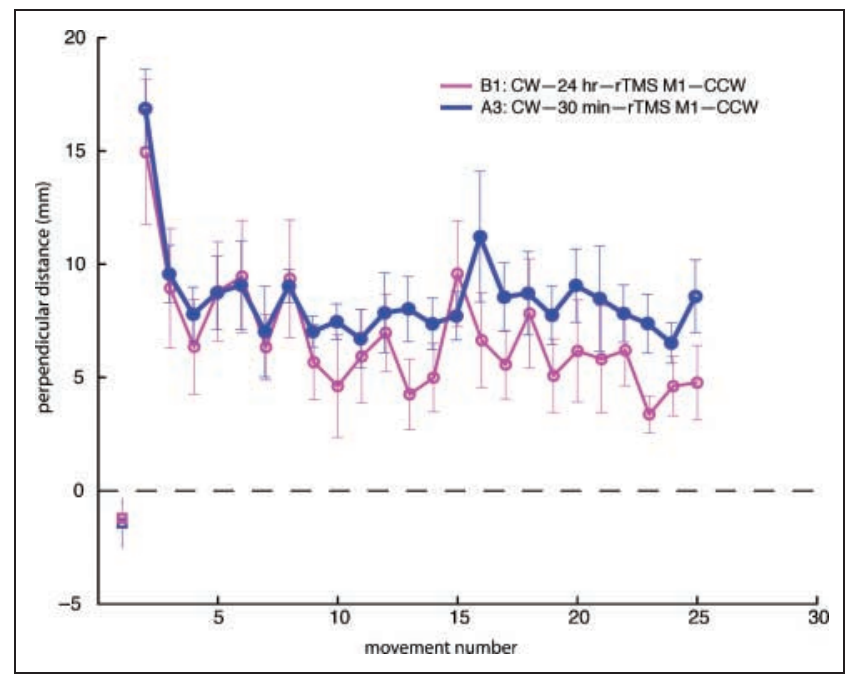

Figure 4. Effects of rTMS on proactive interference following a 30-min or 24-hr delay. Performance in CCW for subjects who previously learned CW followed by a 30-min delay (blue) or a 24-hr delay (magenta) and rTMS over M1. Each point denotes the mean PD over eight movements. Vertical bars correspond to one standard error of the mean. Squares indicate mean PD over the last 16 movements prior to exposure to CCW.

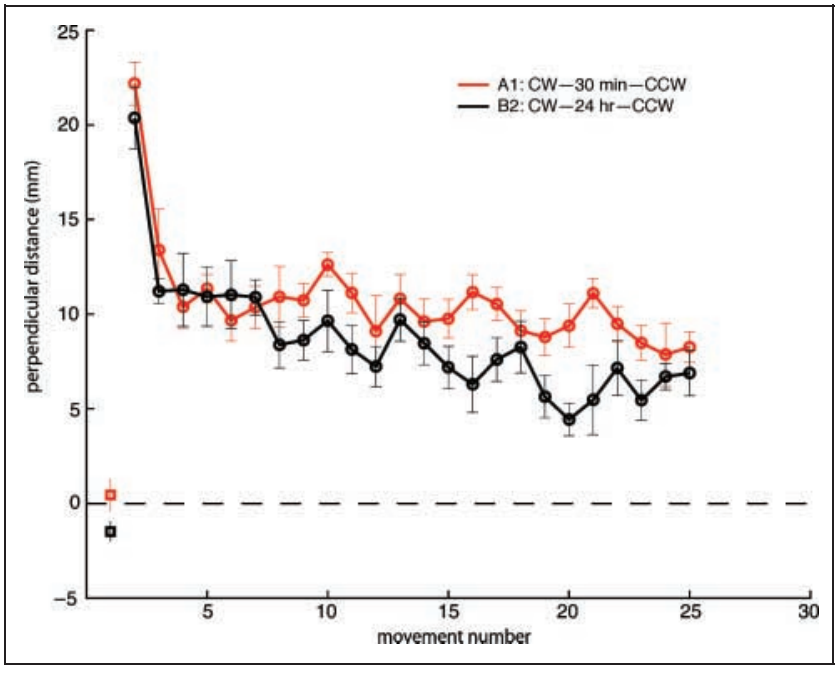

Figure 5. Proactive interference following a 30-min or 24-hr delay. Performance in CCW for subjects who previously learned CW followed by a 30-min delay (red) or a 24-hr delay (black). Each point denotes the mean PD over eight movements. Vertical bars correspond to one standard error of the mean. Squares indicate mean PD over the last 16 movements prior to exposure to CCW.

prior to CCW between subjects who received rTMS after $24 \mathrm{hr}$ (B1) and those who received rTMS after $30 \mathrm{~min}$ (A3). No significant differences were observed $(p=.86$, independent samples $t$ test; Figure 4).

To further explore the behavioral effect of a temporal delay in the absence of rTMS, we assessed proactive interference in another group of subjects who learned CCW $24 \mathrm{hr}$ after having first learned CW and who did not receive rTMS (B2). Initial performance in CCW (mean PD over the first 16 movements) was not significantly different from that of subjects who learned the same sequence of force fields separated by only $30 \mathrm{~min}$ (A1) $(p=.23$, independent samples $t$ test; Figure 5). Together with our rTMS results, these data are not consistent with the idea that for the motor learning paradigm in these studies, consolidation took place over $24 \mathrm{hr}$.

\section{The Effect of rTMS on Retention}

The underlying assumption in the experiments described above is that proactive interference is reduced by rTMS applied to M1 due to disruption of the retention of a first force field. In an additional experiment, we tested this idea directly. We tested for differences in performance of two groups of subjects that learned CCW and, following a 30-min delay, were tested on the same force field. One group received rTMS delivered to M1 during the delay $(\mathrm{C} 2)$; the other group did not receive rTMS (C1). If M1 is involved in the retention of recently learned motor skills, then we would predict that rTMS should result in a decrease in subsequent performance. Initial performance was assessed by computing mean PD 
over the first 16 movements in CCW. Indeed, performance for the group that received rTMS (C2) was significantly worse than that of the corresponding control group (C1) $(p<.05$, independent samples $t$ test; mean $\mathrm{PD}=5.0 \mathrm{~mm}, S E=0.58 \mathrm{~mm}$, and mean $\mathrm{PD}=3.3 \mathrm{~mm}$, $S E=0.34 \mathrm{~mm}$, respectively; Figure $3 \mathrm{~B}$ )

To rule out the possibility that this difference in retention was due to preexisting differences in motor performance at the end of the first learning phase, we tested for differences in movement curvature between the two groups during the last 16 movements prior to the delay. No significant differences were observed ( $p=$ .59, independent samples $t$ test; Figure 3B). Together, these results support the idea that M1 is involved in the retention of recently learned motor skills.

\section{Anatomical and Behavioral Specificity}

The possibility exists that the observed effects of rTMS arose as a nonspecific consequence of brain stimulation rather than as a result of M1 stimulation specifically. To rule this out, we performed two additional control studies with groups of subjects who underwent rTMS delivered to the left superior frontal gyrus (SFG). To rule out nonspecific effects of rTMS on proactive interference, one group of subjects underwent rTMS delivered to SFG during the 30-min delay following learning of CW, prior to CCW (D1). We tested for differences in initial performance in CCW between this group and the group of subjects who did not receive rTMS but were exposed to the same force fields (A1). No significant differences in mean PD over the first 16 movements in CCW were observed between these two groups ( $p=.26$, independent samples $t$ test). To directly assess the anatomical specificity of the effects of rTMS on interference, we also tested for differences in initial performance in CCW between the group receiving rTMS to SFG (D1) and the group receiving rTMS over M1 (A3). Mean PD over the first 16 trials in CCW was significantly lower for the group receiving rTMS over M1 $(p<.05$, independent samples $t$ test; D1: mean $\mathrm{PD}=20.1 \mathrm{~mm}, S E=1.6 \mathrm{~mm}$, and $\mathrm{A} 3$ : mean $\mathrm{PD}=13.3 \mathrm{~mm}, S E=0.91 \mathrm{~mm}$, respectively; Figure 6). More importantly, this effect was not due to preexisting differences prior to $\mathrm{CCW}$; mean $\mathrm{PD}$ over the last 16 trials prior to $\mathrm{CCW}$ was not significantly different between the two groups (D1 vs. $\mathrm{A} 3 ; p=.82$, independent samples $t$ test; Figure 6). Taken together, these results rule out the possibility that the observed effects of rTMS over M1 on proactive interference were due to nonspecific effects of brain stimulation.

To rule out nonspecific effects of rTMS on retention, another group of subjects underwent rTMS delivered to SFG during a 30-min delay following learning of CCW, prior to retesting in CCW (D2). No significant differences in mean PD over the first 16 movements in the CCW retest were observed between these subjects (D2) and the subjects who had the same force fields but did not

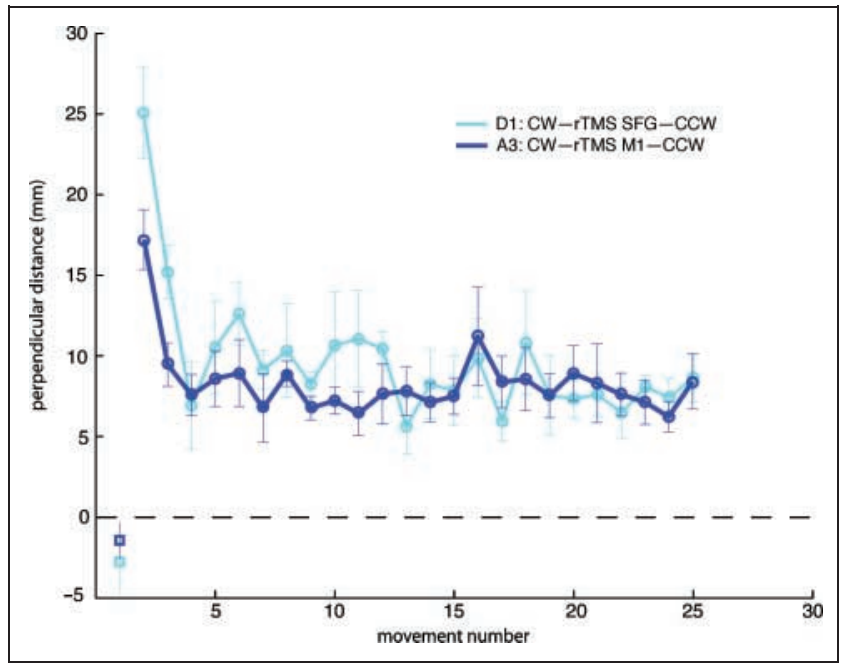

Figure 6. Anatomical specificity of the effects of rTMS on proactive interference. Performance in CCW for subjects who previously learned CW followed by a 30-min delay and rTMS over M1 (blue) or the SFG (cyan). Each point denotes the mean PD over eight movements. Vertical bars correspond to one standard error of the mean. Squares indicate mean PD over the last 16 movements prior to exposure to CCW.

receive rTMS (D2: mean $\mathrm{PD}=2.5 \mathrm{~mm}, S E=0.68 \mathrm{~mm}$; $p=.65$, independent samples $t$ test; Figure 3B). Taken together, these results suggest that like the reduced interference, the reduction in retention we observed following rTMS did not reflect a nonspecific effect of stimulation. Rather, these findings support the idea that M1 plays a critical role in retention and interference (Li et al., 2001; Gandolfo et al., 2000; Classen et al., 1998; Karni et al., 1998; Karni et al., 1995).

Another possibility is that the observed effects of rTMS on movement curvature were due not to a disruption of a neural representation of force field learning, but rather a disturbance in basal motor performance, that is, the more general ability to control rapid movements of the limb. To test this, we compared three measures of basal movement performance for the mean of the first 16 trials in CCW, between subjects who received rTMS during the delay and those who did not. To assess the ability of subjects to produce the appropriate movement speed, we computed mean peak tangential hand velocity. To assess temporal consistency, we computed mean time to peak velocity. Finally, to assess more general spatiotemporal control of trajectory smoothness, we computed root-mean-squared jerk for each hand path. Jerk is the rate of change of acceleration and is a common measure of trajectory smoothness (Flash \& Hogan, 1985). In both the proactive interference experiment and the retention experiment, no significant differences were observed in any of the three measures between subjects receiving rTMS and nonrTMS controls (A3 vs. A1, and C2 vs. C1, respectively) $(p>.11$ in all cases, independent samples $t$ test). 
Following training in NULL, the performance of subjects during the first 16 movements in CCW (A2) did not differ significantly from that of subjects who performed the same training regimen but received rTMS over M1 between the initial training in NULL and subsequent training in CCW (E1) $(p=.18$; split-plot ANOVA; Figure 7). Additionally, the interaction between the withingroups factor (PD, first movement in $\mathrm{A} 2$ and $\mathrm{E} 1 \mathrm{vs}$. two subsequent movements in A2 and E1, respectively) and the between-groups factor (PD, A2 vs. E1) failed to reach significance ( $p=.23$, split-plot ANOVA). This indicates that the learning rate during initial performance in CCW was not affected by M1 and lends further credence to the notion that the observed effects of rTMS over M1 do not reflect a disruption of basal motor performance. There were no preexisting differences in performance between these two groups during the last 16 movements in NULL (A2 vs. E1; $p=.18$, independent samples $t$ test; Figure 7).

\section{DISCUSSION}

The goal of the present study was to examine the relationship between retention and interference in motor learning and its potential neural basis in M1. In an experiment comparing performance during initial exposure and subsequent reexposure to a force field, we found that rTMS delivered to M1 following initial exposure resulted in a decrease in performance during reexposure to the force field. Hence, rTMS over M1

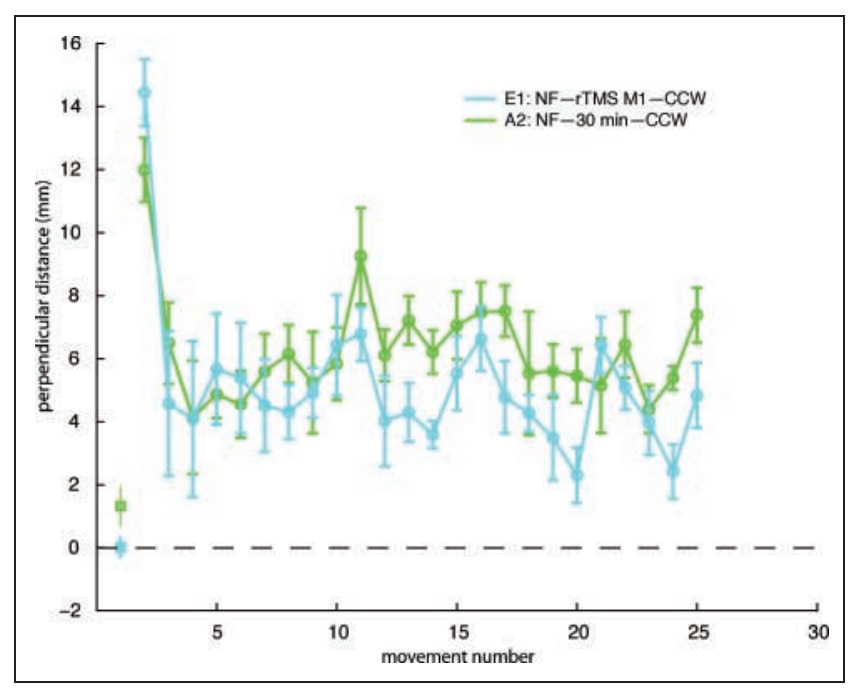

Figure 7. The effect of rTMS on learning rate. Performance in CCW for subjects with no previous training in a force field (green) and subjects with no previous training in a force field followed by rTMS over M1 (cyan). Each point denotes the mean PD over eight movements. Vertical bars correspond to one standard error of the mean. Squares indicate mean PD over the last 16 movements prior to exposure to CCW. disrupted retention (see Figure 3B). In addition, we showed that rTMS over M1 resulted in a reduction in proactive interference (i.e., an improvement in performance) when subjects learned a second force field (see Figure 3A). Taken together, these findings are consistent with the view that proactive interference results from the effects of persisting neural representations of previously learned skills that hinder the ability to encode new motor skills.

We ruled out the possibility that these effects reflect a nonspecific consequence of brain stimulation. When rTMS was delivered to a cortical area in the prefrontal cortex, no effects on performance were observed. We also ruled out the possibility that the observed effects were simply due to a disruption of basal motor performance. First, rTMS had no effects on movement speed, timing, or trajectory smoothness. Second, the effects of rTMS on movement performance were different depending on the specific learning condition. When the learning task following rTMS was the same as the task prior to rTMS, the effect of stimulation was a decrease in performance. In contrast, when rTMS was followed by a new learning task, the effect was an improvement in performance (see Figure $3 \mathrm{~A}$ and $\mathrm{B}$ ).

The present results are consistent with studies that have demonstrated a critical role for $\mathrm{M} 1$ in motor learning (Gribble \& Scott, 2002; Li et al., 2001; Gandolfo et al., 2000; Classen et al., 1998; Karni et al., 1998; Karni et al., 1995), and extend the findings of a recent study that suggests a role for M1 in retention (Muellbacher et al., 2002). In that study, low-frequency rTMS delivered to contralateral M1 resulted in a decrease in performance of a simple, finger pinch task. In a more recent study this finding was replicated, although the authors reported no evidence for an effect of rTMS on the retention of a motor learning task involving finger movements in novel force fields (Baraduc, Lang, Rothwell, \& Wolpert, 2004). The latter finding may appear to be inconsistent with the present results. However, there are important differences between the present study and the experiments reported by Baraduc et al. (2004). Although the task in both studies involved learning to adapt movements performed in a force field, only our study required the use of the entire limb; the task employed by Baraduc et al. involved movements of a single digit. Moreover, the targeted location of rTMS stimulation within M1 was different. In the current study, stimulation was directed at the region representing proximal arm muscles, whereas Baraduc et al. targeted an area corresponding to a finger muscle (first dorsal interosseus). One possibility is that the distributed neural representations associated with the control of multiple segments (Gribble \& Scott, 2002) are more susceptible to disruption from rTMS than the more focal representations governing movements of single digits.

The neurophysiological mechanisms by which interference occurs are not fully understood. Based on electro- 
physiological recordings in M1, it is likely that the same neural ensembles are involved in representing multiple motor skills. Electrophysiological recordings from M1 in monkeys show that the same neurons encode multiple mechanical contexts (Gribble \& Scott, 2002). In studies of motor learning in monkeys, Gandolfo et al. (2000) and Li et al. (2001) showed that tuning curves of single neurons changed after adaptation to a novel force field. Presumably, when these initial changes are inappropriate for a subsequent skill, the persistence of these unsuitable changes interferes with the ability to rapidly generate adjustments in tuning that are appropriate for a new, different skill. Recent studies involving the modulation of various neurotransmitters provide further insights into the neurophysiological basis of M1 plasticity and retention. Butefisch et al. (2000) showed that administration of lorazepam, a $\gamma$-aminobutyric acid (GABA) receptor agonist, reduces M1 plasticity in a thumb movement task. Similarly, Donchin, Sawaki, Madupu, Cohen, and Shadmehr (2002) report that drugs enhancing GABAergic receptor function impaired the acquisition of a novel arm movement skill. Notably, one of the proposed mechanisms by which low-frequency rTMS reduces cortical excitability is the activation of GABAergic interneurons (Gangitano et al., 2002; PascualLeone, Valls-Sole, Wassermann, \& Hallett, 1994). Thus, modulation of GABAergic function is likely one of the mechanisms underlying the effects of rTMS on retention and proactive interference reported here.

In the present study, we did not find evidence in support of the idea that recently acquired motor skills are consolidated into a protected state following dynamic motor learning. We observed a comparable reduction in proactive interference when rTMS was administered $30 \mathrm{~min}$ or $24 \mathrm{hr}$ after learning the first force field. This suggests that the representation of the first force field in M1 was not consolidated within $24 \mathrm{hr}$ but remained vulnerable to disruption. We also noted that for subjects who did not receive rTMS, proactive interference after a 24-hr delay was comparable to that after a 30-min delay. Our findings are inconsistent with those reported by others who concluded that neural representations associated with motor learning become consolidated over the course of several hours (Shadmehr \& Brashers-Krug, 1997; Brashers-Krug et al., 1996). However, the present findings are in agreement with more recent work by Caithness et al. (2004), who conducted six experiments in three laboratories and found no evidence for consolidation using delays as long as one week. Caithness et al. argue for a model of motor learning in which motor memories do not undergo consolidation but can shift between active and inactive states (see Lewis, 1979). There is clearly a need in future work to systematically explore the potential differences among learning tasks and experimental designs that may account for these variations in reported findings.
A number of previous studies have shown that stimulation of a given brain area using TMS can have remote effects on other brain areas (see Paus, 1999, for a review). Thus, it is possible that stimulation of M1 in our study may have resulted in some activity in other brain regions to which $\mathrm{M} 1$ projects. In other words, some component of the observed effects of M1 stimulation on retention and interference may have arisen from interactions of M1 with other sensorimotor brain regions (see Chouinard, Van Der Werf, Leonard, \& Paus, 2003). Indeed, M1 is part of a network of multiple brain regions that are implicated in learning novel motor skills (see Shadmehr \& Wise, 2005; Doyon, Penhune, \& Ungerleider, 2003, for recent reviews). Future studies in which rTMS could be used to disrupt other brain regions known to be involved in the early stages of motor learning and retention (e.g., posterior parietal cortex, premotor cortex) may provide useful insights into the relationship between M1 and these other areas. Regardless of the outcome of such studies, it is important to note that even when interpreting our findings in terms of cortical interactions, M1 would be assigned a crucial functional role. Indeed, there is strong evidence from several electrophysiological and neuroimaging studies demonstrating a critical role of M1 in motor learning and retention (Gribble \& Scott, 2002; Muellbacher et al., 2002; Li et al., 2001; Gandolfo et al., 2000; Classen et al., 1998; Karni et al., 1995).

We have shown here that rTMS of M1 results in two parallel findings, impaired retention and a reduction in proactive interference. Although one cannot rule out the possibility that these two observed effects may be mediated by separate neural mechanisms, the current account linking the two is the more parsimonious one. It is also consistent with other recent empirical findings and theoretical accounts linking interference with the effects of persisting representations of previously learned skills (Bays, Flanagan, \& Wolpert, 2005; Tong, Wolpert, \& Flanagan, 2002; Wigmore et al., 2002; Shadmehr \& Brashers-Krug, 1997; Shadmehr \& Holcomb, 1997).

In summary, our findings support the idea that proactive interference is intimately tied to the retention of recently learned motor skills in M1. They suggest that proactive interference results from the effects of persisting neural representations of recently learned skills that impede the acquisition of new neural representations associated with novel skills.

\section{Acknowledgments}

The authors thank J. Gati and K. Trewartha for technical assistance. This research was supported by grants to P. L. G. and S. K. from the Canadian Institutes of Health Research (CIHR) and the Natural Sciences and Engineering Research Council of Canada (NSERC). P. L. G. was supported by a CIHR New Investigator Award. N. C. was supported by an NSERC postgraduate scholarship. 
Reprint requests should be sent to Paul L. Gribble, Department of Psychology, The University of Western Ontario, London, Ontario, Canada N6A 5C2, or via e-mail: pgribble@uwo.ca.

\section{REFERENCES}

Baraduc, P., Lang, N., Rothwell, J. C., \& Wolpert, D. M. (2004). Consolidation of dynamic motor learning is not disrupted by rTMS of primary motor cortex. Current Biology, 14, 252-256.

Bays, P. M., Flanagan, J. R., \& Wolpert, D. M. (2005). Interference between velocity-dependent and position-dependent force-fields indicates that tasks depending on different kinematic parameters compete for motor working memory. Experimental Brain Research, 163, 400-405.

Brashers-Krug, T., Shadmehr, R., \& Bizzi, E. (1996). Consolidation in human motor memory. Nature, 382, 252-255.

Butefisch, C. M., Davis, B. C., Wise, S. P., Sawaki, L., Kopylev, L., Classen, J., et al. (2000). Mechanisms of use-dependent plasticity in the human motor cortex. Proceedings of the National Academy of Sciences, U.S.A., 97, 3661-3665.

Caithness, G., Osu, R., Bays, P., Chase, H., Klassen, J., Kawato, M., et al. (2004). Failure to consolidate the consolidation theory of learning for sensorimotor adaptation tasks. Journal of Neuroscience, 24, 8662-8671.

Chouinard, P. A., Van Der Werf, Y. D., Leonard, G., \& Paus, T. (2003). Modulating neural networks with transcranial magnetic stimulation applied over the dorsal premotor and primary motor cortices. Journal of Neurophysiology, 90, 1071-1083.

Classen, J., Liepert, J., Wise, S. P., Hallett, M., \& Cohen, L. G. (1998). Rapid plasticity of human cortical movement representation induced by practice. Journal of Neurophysiology, 79, 1117-1123.

Conditt, M. A., Gandolfo, F., \& Mussa-Ivaldi, F. A. (1997). The motor system does not learn the dynamics of the arm by rote memorization of past experience. Journal of Neurophysiology, 78, 554-560.

Di Lazzaro, V., Oliviero, A., Pilato, F., Saturno, E., Dileone, M., Mazzone, P., et al. (2004). The physiological basis of transcranial motor cortex stimulation in conscious humans. Clinical Neurophysiology, 115, 255-266.

Donchin, O., Sawaki, L., Madupu, G., Cohen, L. G., \& Shadmehr, R. (2002). Mechanisms influencing acquisition and recall of motor memories. Journal of Neurophysiology, 88, 2114-2123.

Doyon, J., Penhune, V., \& Ungerleider, L. G. (2003). Distinct contribution of the cortico-striatal and cortico-cerebellar systems to motor skill learning. Neuropsychologia, 41, 252-262.

Flanagan, J. R., \& Wing, A. M. (1997). The role of internal models in motion planning and control: Evidence from grip force adjustments during movements of hand-held loads. Journal of Neuroscience, 17, 1519-1528.

Flash, T., \& Hogan, N. (1985). The coordination of arm movements: An experimentally confirmed mathematical model. Journal of Neuroscience, 5, 1688-1703.

Gandolfo, F., Li, C., Benda, B. J., Schioppa, C. P., \& Bizzi, E. (2000). Cortical correlates of learning in monkeys adapting to a new dynamical environment. Proceedings of the National Academy of Sciences, U.S.A., 97, 2259-2263.
Gangitano, M., Valero-Cabre, A., Tormos, J. M., Mottaghy, F. M., Romero, J. R., \& Pascual-Leone, A. (2002). Modulation of input-output curves by low and high frequency repetitive transcranial magnetic stimulation of the motor cortex. Clinical Neurophysiology, 113, 1249-1257.

Gribble, P. L., \& Scott, S. H. (2002). Overlap of internal models in motor cortex for mechanical loads during reaching. Nature, 417, 938-941.

Karni, A., Meyer, G., Jezzard, P., Adams, M. M., Turner, R., \& Ungerleider, L. G. (1995). Functional MRI evidence for adult motor cortex plasticity during motor skill learning. Nature, 377, 155-158.

Karni, A., Meyer, G., Rey-Hipolito, C., Jezzard, P., Adams, M. M., Turner, R., et al. (1998). The acquisition of skilled motor performance: Fast and slow experience-driven changes in primary motor cortex. Proceedings of the National Academy of Sciences, U.S.A., 95, 861-868.

Lewis, D. J. (1979). Psychobiology of active and inactive memory. Psychological Bulletin, 86, 1054-1083.

Li, C. S., Padoa-Schioppa, C., \& Bizzi, E. (2001). Neuronal correlates of motor performance and motor learning in the primary motor cortex of monkeys adapting to an external force field. Neuron, 30, 593-607.

Muellbacher, W., Ziemann, U., Wissel, J., Dang, N., Kofler, M., Facchini, S., et al. (2002). Early consolidation in human primary motor cortex. Nature, 415, 640-644.

Pascual-Leone, A., Bartres-Faz, D., \& Keenan, J. P. (1999). Transcranial magnetic stimulation: Studying the brainbehaviour relationship by induction of 'virtual lesions.' Philosophical Transactions of the Royal Society of London, Series B, Biological Sciences, 354, 1229-1238.

Pascual-Leone, A., Valls-Sole, J., Wassermann, E. M., \& Hallett, M. (1994). Responses to rapid-rate transcranial magnetic stimulation of the human motor cortex. Brain, 117, 847-858.

Paus, T. (1999). Imaging the brain before, during, and after transcranial magnetic stimulation. Neuropsychologia, 37, 219-224.

Shadmehr, R., \& Brashers-Krug, T. (1997). Functional stages in the formation of human long-term motor memory. Journal of Neuroscience, 17, 409-419.

Shadmehr, R., \& Holcomb, H. H. (1997). Neural correlates of motor memory consolidation. Science, 277, 821-825.

Shadmehr, R., \& Mussa-Ivaldi, F. A. (1994). Adaptive representation of dynamics during learning of a motor task. Journal of Neuroscience, 14, 3208-3224.

Shadmehr, R., \& Wise, S. P. (2005). The computational neurobiology of reaching and pointing: A foundation for motor learning. Cambridge: MIT Press.

Thoroughman, K. A., \& Shadmehr, R. (1999). Electromyographic correlates of learning an internal model of reaching movements. Journal of Neuroscience, 19, 8573-8588.

Tong, C., Wolpert, D. M., \& Flanagan, J. R. (2002). Kinematics and dynamics are not represented independently in motor working memory: Evidence from an interference study. Journal of Neuroscience, 22, 1108-1113.

Wassermann, E. M., Grafman, J., Berry, C., Hollnagel, C., Wild, K., Clark, K., et al. (1996). Use and safety of a new repetitive transcranial magnetic stimulator. Electroencephalography and Clinical Neurophysiology, 101, 412-417.

Wigmore, V., Tong, C., \& Flanagan, J. R. (2002). Visuomotor rotations of varying size and direction compete for a single internal model in motor working memory. Journal of Experimental Psychology: Human Perception and Performance, 28, 447-457. 